\title{
Development and validation of a $25-G e n e$ Panel urine test for prostate cancer diagnosis and potential treatment follow-up
}

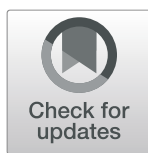

Heather Johnson', Jinan Guo ${ }^{2,3}$, Xuhui Zhang ${ }^{4}$, Heqiu Zhang ${ }^{4}$, Athanasios Simoulis ${ }^{5}$, Alan H. B. Wu', Taolin Xia ${ }^{7}$, Fei Li ${ }^{8}$, Wanlong Tan ${ }^{8}$, Allan Johnson ${ }^{9}$, Nishtman Dizeyi ${ }^{10}$, Per-Anders Abrahamsson ${ }^{10}$, Lukas Kenner ${ }^{11}$, Xiaoyan Feng ${ }^{4}$, Chang Zou ${ }^{3+}$, Kefeng Xiao ${ }^{2,3+}$, Jenny L. Persson ${ }^{12,13,14^{*}+}$ and Lingwu Chen ${ }^{15^{*}+}$

\begin{abstract}
Background: Heterogeneity of prostate cancer (PCa) contributes to inaccurate cancer screening and diagnosis, unnecessary biopsies, and overtreatment. We intended to develop non-invasive urine tests for accurate PCa diagnosis to avoid unnecessary biopsies.

Methods: Using a machine learning program, we identified a 25-Gene Panel classifier for distinguishing PCa and benign prostate. A non-invasive test using pre-biopsy urine samples collected without digital rectal examination (DRE) was used to measure gene expression of the panel using CDNA preamplification followed by real-time qRTPCR. The 25-Gene Panel urine test was validated in independent multi-center retrospective and prospective studies. The diagnostic performance of the test was assessed against the pathological diagnosis from biopsy by discriminant analysis. Uni- and multivariate logistic regression analysis was performed to assess its diagnostic improvement over PSA and risk factors. In addition, the 25-Gene Panel urine test was used to identify clinically significant PCa. Furthermore, the 25-Gene Panel urine test was assessed in a subset of patients to examine if cancer was detected after prostatectomy.
\end{abstract}

Results: The 25-Gene Panel urine test accurately detected cancer and benign prostate with AUC of $0.946(95 \% \mathrm{Cl}$ 0.963-0.929) in the retrospective cohort $(n=614)$, AUC of $0.901(0.929-0.873)$ in the prospective cohort $(n=396)$, and AUC of $0.936(0.956-0.916)$ in the large combination cohort $(n=1010)$. It greatly improved diagnostic accuracy over PSA and risk factors $(p<0.0001)$. When it was combined with PSA, the AUC increased to $0.961(0.980-0.942)$. Importantly, the 25-Gene Panel urine test was able to accurately identify clinically significant and insignificant PCa with AUC of 0.928 ( $95 \% \mathrm{Cl} 0.947-0.909)$ in the combination cohort $(n=727)$. In addition, it was able to show the absence of cancer after prostatectomy with high accuracy.

(Continued on next page)

\footnotetext{
*Correspondence: jenny.persson@umu.se; chenlwu@mail.sysu.edu.cn

${ }^{\dagger}$ Chang Zou, Kefeng Xiao, Jenny L. Persson, and Lingwu Chen contributed equally as senior authors.

${ }^{12}$ Department of Molecular Biology, Umeå University, 90187 Umeå, Sweden

${ }^{15}$ Department of Urology, The First Affiliated Hospital of Sun Yat-Sen University, Guangzhou 510080, Guangdong, China

Full list of author information is available at the end of the article
}

(c) The Author(s). 2020 Open Access This article is licensed under a Creative Commons Attribution 4.0 International License, which permits use, sharing, adaptation, distribution and reproduction in any medium or format, as long as you give appropriate credit to the original author(s) and the source, provide a link to the Creative Commons licence, and indicate if changes were made. The images or other third party material in this article are included in the article's Creative Commons licence, unless indicated otherwise in a credit line to the material. If material is not included in the article's Creative Commons licence and your intended use is not permitted by statutory regulation or exceeds the permitted use, you will need to obtain permission directly from the copyright holder. To view a copy of this licence, visit http://creativecommons.org/licenses/by/4.0/. The Creative Commons Public Domain Dedication waiver (http://creativecommons.org/publicdomain/zero/1.0/) applies to the data made available in this article, unless otherwise stated in a credit line to the data. 
(Continued from previous page)

Conclusions: The 25-Gene Panel urine test is the first highly accurate and non-invasive liquid biopsy method without DRE for PCa diagnosis. In clinical practice, it may be used for identifying patients in need of biopsy for cancer diagnosis and patients with clinically significant cancer for immediate treatment, and potentially assisting cancer treatment follow-up.

Keywords: Prostate cancer, Prostate cancer diagnosis, Clinically significant prostate cancer, Prostate cancer treatment follow-up, Gene Panel, Urine test

\section{Background}

Prostate cancer (PCa) is the second most prevalent cancer and a leading cause of cancer-related death [1]. Needle biopsy is a standard method for PCa diagnosis, yet it is invasive and associated with complications and missing lesions [2]. Prostate-specific antigen (PSA) is a widely used PCa screening test, yet with moderate sensitivity and very low specificity $(<30 \%)$, resulting in $>70 \%$ false positive rate and many unnecessary biopsies [2]. Although tests using PSA isoforms/analogs have been developed, their improvement on accuracy is limited [2, 3]. For clinically meaningful PCa diagnosis, it is important to identify patients with clinically significant cancer. Although the new tools such as magnetic resonance imaging (MRI) and multiparametric MRI targeted biopsy have been used to identify patients with clinically significant PCa, these methods have limited accuracy [4-6].

During tumorigenesis, PCa cells are exfoliated from the prostate and released into the urine [7], making urine a readily available source to detect prostate-specific biomarkers for diagnosis and prognosis. Although many urine biomarkers have been identified and used individually or in combination for diagnosis, none of them has sensitivity and specificity both above $90 \%$ and AUC above 0.9. Most studies tested in $<300$ samples. All of them use urine collected after digital rectal examination (DRE), which is invasive and uncomfortable for patients [2, 6, 8-12]. In addition, with very low specificity of the PSA test for cancer diagnosis and limitation of imaging technologies to identify residual cancer lesions after treatment, no accurate test is available to assess efficacy and outcome of PCa treatment such as prostatectomy. Yet it is crucial to accurately measure treatment outcome to assist treatment decision-making, such as assessing if residual cancer lesion remains after prostatectomy to determine the necessity of subsequent treatment, leading to improved cancer treatment and reduced mortality [13, 14]. Therefore, it is of great clinical significance to develop better tests for these unmet medical needs.

$\mathrm{PCa}$ is a cancer with a high degree of heterogeneity. Many gene alterations contribute to cancer tumorigenesis, progression, recurrence, and metastasis [15]. Thus, it is necessary to combine multiple biomarkers involved in these processes.
We therefore developed a novel 25-Gene Panel urine test for PCa diagnosis and potential treatment follow-up. We showed that the test was robust with high accuracy in two independent, multi-center studies.

\section{Methods}

\section{Retrospective and prospective studies}

A multi-center retrospective study was approved by the Institutional Review Board (IRB) of San Francisco General Hospital (San Francisco, USA) (IRB \# 15-15816) to collect and test archived urine sediments to identify and validate urine biomarkers for $\mathrm{PCa}$ diagnosis. The prospectively designed, retrospectively collected pre-biopsy urine samples were randomly picked from sample archives at Cooperative Human Tissue Network (CHTN) Southern Division (patients in the USA) and Indivumed $\mathrm{GmbH}$ (patients in Germany). The urine samples were collected from patients with elevated PSA scheduled for biopsy for cancer diagnosis from July 2004 to November 2014 with prior ethical approval and patient consent for future studies. A multi-center prospective study for urine biomarkers was approved by IRB of Shenzhen People's Hospital (Shenzhen, China) (Study Number P2014006) to collect pre-biopsy fresh urine samples from patients treated at seven hospitals collaborated in the study with patient consent, including Shenzhen People's Hospital, The First Affiliated Hospital of Sun Yat-Sen University, Peking University First Hospital, Foshan First People's Hospital, Nanfang Hospital at Southern Medical University, Peking University Shenzhen Hospital, and The Second People's Hospital of Shenzhen. The urine samples were collected consecutively from patients with elevated PSA scheduled for biopsy in the participating hospitals. Both studies used the same patient inclusion criteria of age at 18-85, with histopathological diagnosis of $\mathrm{PCa}, \mathrm{BPH}$, or prostatitis from biopsy, and without treatment of PCa drugs or 5-alpha reductase inhibitors prior to urine collection. The exclusion criteria included having prostatectomy or treatment with PCa drugs or 5alpha reductase inhibitors before urine collection. In addition, ten patients undergoing prostatectomy were recruited to collect urine samples several days before and after surgery. The pathological diagnosis of $\mathrm{PCa}$ in both 
retrospective and prospective studies was performed by using standard needle biopsy with consistent procedures. The pathological diagnosis of clinically significant or insignificant PCa was defined based on PCa risk stratification guidelines from the National Comprehensive Cancer Network (NCCN) with modifications. The clinically significant $\mathrm{PCa}$ patients were classified as meeting any of the following criteria: Gleason score $>7$, Gleason score $4+3=7$, cancer staging $\geq \mathrm{T} 3$, PSA $>20 \mathrm{ng} / \mathrm{mL}$ at diagnosis, biochemical recurrence after prostatectomy during the follow-up period, or cancer metastasis at diagnosis or during the follow-up period. The rest of the patients were classified as clinically insignificant PCa. All samples were de-identified and coded with patient numbers to protect patient privacy following the Health Insurance Portability and Accountability Act guidelines. Urine samples from 665 patients were received with 51 excluded in the retrospective cohort and urine samples from 411 patients were received with 15 excluded in the prospective cohort respectively, due to the lack of pathology report, diagnosis uncertainty, or low/no gene expression detected.

\section{Urine processing and quantification of gene expression}

For the retrospective study, 10-15 $\mathrm{mL}$ urine samples were collected without digital rectal examination (DRE) and the urine pellet was flash-frozen and stored at $80^{\circ} \mathrm{C}$. For the prospective study, $15-45 \mathrm{~mL}$ urine without DRE was collected in the presence of $5 \mathrm{~mL}$ DNA/ RNA preservative AssayAssure (Thermo Fisher Scientific, Waltham, MA, USA) or U-Preserve (Hao Rui Jia Biotech Ltd., Beijing, China), stored at $4{ }^{\circ} \mathrm{C}$, and processed within 7 days. The urine pellet obtained after centrifugation at $1000 \times g$ for $10 \mathrm{~min}$ was washed with phosphate-buffered saline followed by a second centrifugation at $1000 \times g$ for $10 \mathrm{~min}$. The cell pellet was processed for RNA purification or immediately frozen on dry ice and stored at $-80^{\circ} \mathrm{C}$. A detailed procedure of gene expression quantification is listed in Additional file 1: Methods.

\section{Prostate tissue specimen cohort}

The GSE17951 prostate tissue specimen cohort includes quantitative mRNA expression data of $\mathrm{PCa}$ and benign prostate specimens obtained from Affymetrix U133Plus2 array $[16,17]$. The PCa tissues $(n=56)$ in the cohort were collected from patient biopsy specimens, and the benign prostate tissues $(n=98)$ were obtained from prostate autopsy specimens of patients with benign disease. The gene expression levels of the 25 genes in the panel were obtained from the database and normalized with beta-actin expression level.

\section{Data analysis and algorithm for cancer diagnosis}

All data analysis and diagnosis by the 25-Gene Panel were performed blindly without prior knowledge of patient information. The gene expression data was downloaded and first analyzed with ABI Quantstudio 6 software (Thermo Fisher Scientific, Waltham, MA, USA). The mRNA expression level of the housekeeping gene beta-actin was measured in each urine sample and used for gene expression normalization to control variation of cDNA quantity in the urine samples. The cycle threshold $(\mathrm{Ct})$ value of each gene in the panel was divided by the $\mathrm{Ct}$ value of the beta-actin and then multiplied by 1000 as the normalized gene expression value $(\mathrm{CtS}=\mathrm{Ct}($ sample $) / \mathrm{Ct}($ actin $) \times 1000)$. For each gene, the average $\mathrm{Ct}$ value from triplicate PCR was used. For the diagnosis of cancer by the 25-Gene Panel, the relative $\mathrm{Ct}$ $(\mathrm{CtS})$ values of the 25 genes in the panel were used to generate a classification score (diagnostic $D$ score).

For cancer diagnosis in both retrospective and prospective cohorts, each sample was diagnosed using the Diagnosis Algorithm as shown below:

$$
\begin{aligned}
C_{\mathrm{PCa}}= & A_{\mathrm{PCa}}+\mathrm{CtS}_{1} * X_{1}+\mathrm{CtS}_{2} * X_{2 \ldots}+\mathrm{CtS}_{25} * X_{25} \\
& +\mathrm{CtS}_{1} * \mathrm{CtS}_{1} * X_{1 * 1}+\mathrm{CtS}_{1} * \mathrm{CtS}_{2} * X_{1 * 2 \ldots} \\
& +\mathrm{CtS}_{1} * \mathrm{CtS}_{25} * X_{1 * 25}+\mathrm{CtS}_{2} * \mathrm{CtS}_{2} * X_{2 * 2 \ldots} \\
& +\mathrm{CtS}_{2} * \mathrm{CtS}_{25} * X_{2 * 25 \ldots}+\mathrm{CtS}_{25} * \mathrm{CtS}_{25} * X_{25 * 25} \\
C_{\mathrm{Non}}= & B_{\mathrm{Non}}+\mathrm{CtS}_{1} * Y_{1}+\mathrm{CtS}_{2} * Y_{2 \ldots}+\mathrm{CtS}_{25} * Y_{25} \\
& +\mathrm{CtS}_{1} * \mathrm{CtS}_{1} * Y_{1 * 1}+\mathrm{CtS}_{1} * \mathrm{CtS}_{2} * Y_{1 * 2 \ldots} \\
& +\mathrm{CtS}_{1} * \mathrm{CtS}_{25} * Y_{1 * 25}+\mathrm{CtS}_{2} * \mathrm{CtS}_{2} * Y_{2 * 2 \ldots} \ldots \\
& +\mathrm{CtS}_{2} * \mathrm{CtS}_{25} * Y_{2 * 25 \ldots}+\mathrm{CtS}_{25} * \mathrm{CtS}_{25} * Y_{25 * 25}
\end{aligned}
$$

Diagnostic $D$ score $=C_{\mathrm{PCa}}-C_{\mathrm{Non}}$

Whereas $A_{\mathrm{PCa}}$ is the PCa constant, $B_{\mathrm{Non}}$ is the nonPCa constant, $\mathrm{CtS}_{1}$ through $\mathrm{CtS}_{25}$ are $\mathrm{CtS}$ values of gene 1 through gene 25, $X_{1}$ through $X_{25}$ are PCa regression coefficients of gene 1 through gene 25, $X_{1^{* 1} 1}$ through $X_{25 * 25}$ are gene 1 and gene 1 cross PCa regression coefficients through gene 25 and gene 25 cross PCa regression coefficients, $Y_{1}$ through $Y_{25}$ are non-PCa regression coefficients of gene 1 through gene 25, and $Y_{1^{*} 1}$ through $Y_{25 * 25}$ are gene 1 and gene 1 cross non-PCa regression coefficients through gene 25 and gene 25 cross non-PCa regression coefficients. The sample was diagnosed to be PCa when the diagnostic $D$ score was $>0$, whereas the sample was diagnosed to be benign prostate (non-PCa) when the diagnostic $D$ score was $\leq 0$.

\section{Statistical analysis}

To generate an algorithm for diagnosing urine samples as PCa or benign prostate (Diagnosis Algorithm), discriminant analysis was performed to test the association between pathological diagnosis and $\mathrm{CtS}$ values of the 25 genes in the panel using a statistical software program XLSTAT (Addinsoft, Paris, France). The diagnosis of all 
the samples by the algorithm was compared to their pathological diagnosis to assess diagnostic performance by calculating sensitivity, specificity, positive predictive value, negative predictive value, odds ratio, and their respective $95 \%$ confidence intervals. The receiver operating characteristic curve was plotted and the area under the curve (AUC) with its 95\% confidence interval was calculated. To further validate the 25-Gene Panel in the combination cohort, the leave-one-out cross-validation analysis was performed to generate regression coefficients to determine the classification of each sample by the 25-Gene Panel, which was then compared with the pathological diagnosis of each sample to calculate the diagnostic performance of cross-validation using XLSTAT. In addition, univariate and multivariate logistic regression analyses were conducted to compare the diagnostic performance of pre-biopsy PSA, pre-biopsy PSA at the cutoff value of $4 \mathrm{ng} / \mathrm{mL}$, patient age, PCa family history, the 25-Gene Panel urine test, and their combinations.

\section{Results}

\section{Non-invasive urine test}

Current urine tests for $\mathrm{PCa}$ diagnosis and prognosis rely on DRE before urine collection to enrich prostate cells in the urine, yet the procedure is uncomfortable and invasive for patients and requires a physician to perform. To develop a non-invasive urine test to measure gene expression of biomarkers, we employed a modified method of cDNA preamplification before real-time qRTPCR [18] and showed that it improved quantification of gene expression in urine collected without DRE that contained fewer prostate cells. We detected mRNA expression of the genes with significantly increased sensitivity by $\sim 10 \mathrm{Ct}$ units without changing the relative gene expression values $(\Delta \mathrm{Ct})$ (Additional file 2: Table $\mathrm{S} 1)$. The $\Delta \mathrm{Ct}$ values were similar in the urine samples collected from the same patients with and without DRE (Additional file 2: Table S2), the urine with and without DRE had similar diagnostic $D$ score, and the diagnosis of the urine with or without DRE was the same (Table 1). With the help of DNA/RNA preservative, urine can be collected without DRE or physician's involvement and stored or shipped at room temperature within a week. Our data demonstrated that the new method developed in the study is robust and can be used to quantify biomarker gene expression in urine samples without DRE, making it a valid and much improved liquid biopsy method in clinical practice.

\section{Development of the 25-Gene Panel classifier}

In a previous study, we identified a series of biomarker candidates involved in cell proliferation, survival, migration, tumorigenesis, cancer invasion, and metastasis with differential gene expression in $\mathrm{PCa}$ and benign prostate tissue specimens $[19,20]$. To develop a gene panel for cancer diagnosis with high diagnostic accuracy, we used a random forest machine learning program $[21,22]$ combined with a discriminant analysis classification test to screen mRNA expression profiles of the biomarker candidates in $\mathrm{PCa}$ and benign prostate specimens in large cohorts obtained from Gene Expression Omnibus (GEO) database. The diagnosis of the specimens by various panels combining the candidate biomarkers was compared to the pathological diagnosis of the specimens to assess the diagnostic performance of the panels to distinguish $\mathrm{PCa}$ and benign prostate, which included diagnostic parameters of sensitivity, specificity, odds ratio, and AUC. A 25-Gene Panel consisting of HIF1A, FGFR1, BIRC5, AMACR, CRISP3, FN1, HPN, MYO6, PSCA, PMP22, GOLM1, LMTK2, EZH2, GSTP1, PCA3, VEGFA, CST3, PTEN, PIP5K1A, CDK1, TMPRSS2, ANXA3, CCNA1, CCND1, and $K L K 3$ was discovered to have the highest diagnostic accuracy to distinguish cancer lesions from benign prostate (Additional file 2: Table S3). We found that subtracting any one or more genes from the panel would lower the diagnostic accuracy, such as lowered sensitivity, specificity, and AUC. This showed that all genes in the panel contribute significantly to the diagnostic algorithm.

\section{The 25-Gene Panel urine test for cancer diagnosis}

We examined if the 25-Gene Panel identified above can be used for cancer diagnosis using urine samples

Table 1 Diagnosis of urine samples collected with and without DRE

\begin{tabular}{|c|c|c|c|c|c|c|}
\hline & D score-DRE-urine & $D$ score-DRE+urine & SD & SD/mean (\%) & Diagnosis-DRE-urine & Diagnosis-DRE+urine \\
\hline Patient 1 & 30.7 & 31.9 & 0.8 & 2.5 & PCa & $\mathrm{PCa}$ \\
\hline Patient 2 & 30.4 & 30.1 & 0.3 & 0.8 & PCa & PCa \\
\hline Patient 3 & 30.1 & 30.6 & 0.4 & 1.2 & PCa & PCa \\
\hline Patient 4 & 35.0 & 32.9 & 1.5 & 4.3 & PCa & $\mathrm{PCa}$ \\
\hline Patient 5 & 30.5 & 29.9 & 0.4 & 1.4 & PCa & PCa \\
\hline
\end{tabular}

$D R E$ digital rectal examination, $D$ score-DRE-urine diagnostic $D$ score of the urine sample collected without DRE, $D$ score-DRE+urine diagnostic $D$ score of the urine sample collected after DRE, diagnosis-DRE-urine diagnosis of the urine sample collected without DRE, diagnosis-DRE+urine diagnosis of the urine sample collected after DRE 


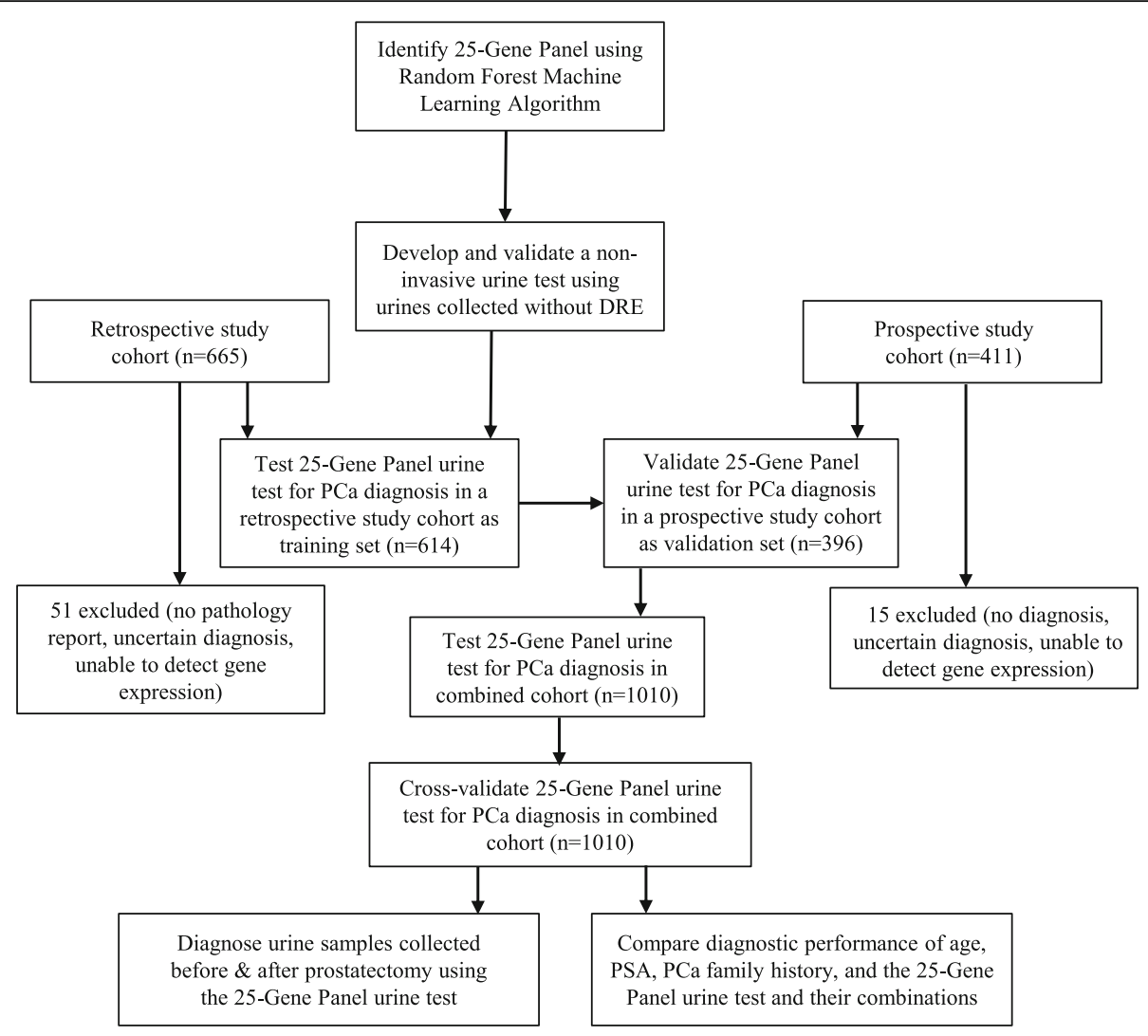

Fig. 1 Study design

collected without DRE (Fig. 1). We conducted independent, multi-center retrospective and multi-center prospective studies to collect pre-biopsy urine samples and used the 25-Gene Panel as a classifier to distinguish $\mathrm{PCa}$ and benign prostate for diagnosis. The study population in both cohorts represents patients in real clinical practice as they are patients who underwent routine cancer diagnosis using standard PSA and biopsy in the participating hospitals. The end point of the study was to assess the diagnostic performance of the 25-Gene Panel urine test and its improvement over the known clinical parameters for $\mathrm{PCa}$ diagnosis. The patient characteristics and clinical parameters are illustrated based on the standard clinical practice [23] as shown in Table 2.

We successfully quantified mRNA expression of each biomarker in the 25-Gene Panel using preamplification of cDNA purified from urine pellets followed by real-time qRT-PCR. The retrospective cohort $(n=614)$ was used as a training set to create the Diagnosis Algorithm, which combined the mRNA expression quantity of the biomarkers in the panel for classification of the urine sample as $\mathrm{PCa}$ or benign prostate. Such diagnosis was then compared to the pathological diagnosis from biopsy to calculate the diagnostic performance of the 25-Gene Panel urine test.
As shown in Table 3 and Fig. 2a, the 25-Gene Panel was capable of distinguishing PCa from benign prostate (non-PCa) with high sensitivity of $92.5 \%$ (95\% CI 94.8 90.2\%), specificity of $91.5 \%$ (95\% CI $97.1-85.9 \%$ ), odds ratio of 132.6 (95\% CI 293.5-59.9), and AUC of 0.946 (95\% CI 0.963-0.929).

We then used an independent multi-center prospective cohort $(n=396)$ as a validation set to assess the diagnostic accuracy of the 25-Gene Panel urine test. The result showed sensitivity of $85.0 \%$ (95\% CI $89.9-80.2 \%$ ), specificity of $94.7 \%$ (95\% CI $97.9-91.5 \%$ ), odds ratio of 101.6 (95\% CI 213.5-48.4), and AUC of 0.901 (95\% CI 0.929-0.873) (Table 3 and Fig. 2b). The diagnostic performance was further validated by combining the retrospective $(n=614)$ and prospective $(n=396)$ cohorts, which used the same inclusion and exclusion criteria to enroll patients and collected urine samples without DRE, to form a combination cohort of 1010 patients with 283 benign prostate $(28.0 \%)$ and $727 \mathrm{PCa}(72.0 \%)$. The 25Gene Panel showed high sensitivity of $90.4 \%$ (95\% CI 92.5-88.2\%), specificity of $93.6 \%$ (95\% CI $96.5-90.8 \%$ ), odds ratio of 138.2 (95\% CI 236.5-80.8), and AUC of 0.936 (95\% CI 0.956-0.916) (Table 3 and Fig. 2c). Crossvalidation of the 25-Gene Panel urine test in the combination cohort generated similarly accurate diagnostic 
Table 2 Patient characteristics

\begin{tabular}{|c|c|c|c|c|c|c|}
\hline & \multicolumn{2}{|c|}{ Retrospective cohort } & \multicolumn{2}{|c|}{ Prospective cohort } & \multicolumn{2}{|c|}{ Combination cohort } \\
\hline & Non-PCa & $\mathrm{PCa}$ & Non-PCa & $\mathrm{PCa}$ & Non-PCa & $\mathrm{PCa}$ \\
\hline Patients (\%) & $94(15.3 \%)$ & $520(84.7 \%)$ & $189(47.7 \%)$ & $207(52.3 \%)$ & $283(28.0 \%)$ & $727(72.0 \%)$ \\
\hline Mean age (year) & $64(41-84)$ & $64(45-78)$ & $69(45-86)$ & 69 (39-88) & $68(41-86)$ & $65(39-88)$ \\
\hline Patients with other cancers (\%) & $1(1.1 \%)$ & $4(0.8 \%)$ & $2(1.1 \%)$ & $1(0.5 \%)$ & $3(1.1 \%)$ & $5(0.7 \%)$ \\
\hline \multicolumn{7}{|l|}{ Gleason score (\%) } \\
\hline Group 1: $\leq 6(\leq 3+3)$ & NA & $124(23.8 \%)$ & NA & $39(18.8 \%)$ & NA & $163(22.4 \%)$ \\
\hline Group 2: $7(3+4)$ & NA & $218(41.9 \%)$ & NA & $54(26.1 \%)$ & NA & $272(37.4 \%)$ \\
\hline Group 3: $7(4+3)$ & NA & $136(26.2 \%)$ & NA & $55(26.6 \%)$ & NA & $191(26.3 \%)$ \\
\hline Group 4: $8(4+4,3+5,5+3)$ & NA & $17(3.3 \%)$ & NA & $30(14.5 \%)$ & NA & $47(6.5 \%)$ \\
\hline Group 5: 9 or $10(4+5,5+4$, or $5+5)$ & NA & $25(4.8 \%)$ & NA & $29(14.0 \%)$ & NA & $54(7.4 \%)$ \\
\hline Mean PSA (ng/mL) & 10.1 & 6.1 & 10.6 & 67.9 & 10.51 & 65.0 \\
\hline
\end{tabular}

measures (Table 3 and Fig. 2d), further proving its accuracy in cancer diagnosis. These results from independent multi-center studies have clearly demonstrated the 25-Gene Panel urine test as an accurate tool to distinguish PCa and benign prostate. This suggests that the non-invasive and accurate urine test can be used to aid PCa diagnosis so only patients diagnosed to have PCa by the 25-Gene Panel urine test need to undergo biopsy to confirm the diagnosis.

\section{Comparison of the diagnostic performance of the 25- Gene Panel urine test with PSA and risk factors}

Since PSA has been widely used as a PCa screening test, and age and $\mathrm{PCa}$ family history are risk factors for cancer, we compared the diagnostic performance of prebiopsy PSA, PSA at $4 \mathrm{ng} / \mathrm{mL}$ cutoff value (commonly used cutoff for further testing in PCa screening) (PSA4 ), age, and $\mathrm{PCa}$ family history (FH) with the 25-Gene Panel urine test (25-Gene) in patients from the combination cohort who had PSA test result or family history information. The patient cohort with PSA test result (referred as PSA Cohort) $(n=411)$ did not overlap with the patient cohort with family history information (referred as FH Cohort) $(n=451)$; thus, PSA and PSA-4 were assessed in the PSA Cohort while FH was assessed in the FH Cohort. Age and the 25-Gene Panel urine test were assessed in both PSA Cohort and FH Cohort. The 25-Gene Panel urine test had much higher accuracy in distinguishing $\mathrm{PCa}$ and benign prostate than PSA, PSA4 , age, and $\mathrm{FH}$ as shown by their respective $p$ value, odds ratio, and AUC in univariate logistic regression analysis $(p<0.0001)$ (Table 4$)$. PSA at $4 \mathrm{ng} / \mathrm{mL}$ cutoff is widely used in cancer screening, yet it had much lower specificity and AUC than the 25-Gene Panel urine test (30.2\% vs $93.2 \%$ and 0.588 vs 0.939 , respectively) (Table 5 ). This result demonstrated that the 25-Gene Panel urine test had superior diagnostic performance than PSA at $4 \mathrm{ng} /$ $\mathrm{mL}$, with greatly improved diagnostic specificity. Each year, more than 700,000 unnecessary negative biopsies were performed in the USA due to $\sim 70 \%$ false positive rate of PSA at $4 \mathrm{ng} / \mathrm{mL}$ in the cancer screening test [24]. If the 25-Gene Panel urine test was used after the PSA

Table 3 Diagnostic performance of the 25-Gene Panel urine test in a retrospective training cohort $(n=614)$, a prospective validation cohort $(n=396)$, a combination cohort $(n=1010)$, and cross-validation of the combination cohort $(n=1010)$

\begin{tabular}{|c|c|c|c|c|c|c|c|c|c|c|c|c|}
\hline & \multicolumn{3}{|c|}{ Retrospective cohort } & \multicolumn{3}{|c|}{ Prospective cohort } & \multicolumn{3}{|c|}{ Combination cohort } & \multicolumn{3}{|c|}{ Cross-validation } \\
\hline & Positive & Negative & $\overline{\text { Total }}$ & Positive & Negative & $\overline{\text { Total }}$ & Positive & Negative & $\overline{\text { Total }}$ & Positive & Negative & Total \\
\hline PCa & 481 & 39 & 520 & 176 & 31 & 207 & 657 & 70 & 727 & 644 & 83 & 727 \\
\hline Non-PCa & 8 & 86 & 94 & 10 & 179 & 189 & 18 & 265 & 283 & 27 & 256 & 283 \\
\hline Total & 489 & 125 & 614 & 186 & 210 & 396 & 675 & 335 & 1010 & 671 & 339 & 1010 \\
\hline Sensitivity (95\% Cl) & \multicolumn{3}{|c|}{$92.5 \%(94.8-90.2 \%)$} & \multicolumn{3}{|c|}{$85.0 \%(89.9-80.2 \%)$} & \multicolumn{3}{|c|}{$90.4 \%(92.5-88.2 \%)$} & \multicolumn{3}{|c|}{$88.6 \%(90.9-86.3 \%)$} \\
\hline Specificity (95\% Cl) & \multicolumn{3}{|c|}{$91.5 \%(97.1-85.9 \%)$} & \multicolumn{3}{|c|}{$94.7 \%$ (97.9-91.5\%) } & \multicolumn{3}{|c|}{$93.6 \%(96.5-90.8 \%)$} & \multicolumn{3}{|c|}{$90.5 \%$ (93.9-87.0\%) } \\
\hline PPV $(95 \%$ Cl) & \multicolumn{3}{|c|}{$98.4 \%$ (99.5-97.2\%) } & \multicolumn{3}{|c|}{$94.6 \%$ (97.9-91.4\%) } & \multicolumn{3}{|c|}{$97.3 \%(98.6-96.1 \%)$} & \multicolumn{3}{|c|}{$96.0 \%(97.5-94.5 \%)$} \\
\hline NPV $(95 \% \mathrm{Cl})$ & \multicolumn{3}{|c|}{$68.8 \%(76.9-60.7 \%)$} & \multicolumn{3}{|c|}{$85.2 \%(90.0-80.4 \%)$} & \multicolumn{3}{|c|}{$79.1 \%(83.5-74.8 \%)$} & \multicolumn{3}{|c|}{$75.5 \%(80.1-70.9 \%)$} \\
\hline Odds ratio $(95 \% \mathrm{Cl})$ & \multicolumn{3}{|c|}{$132.6(293.5-59.9)$} & \multicolumn{3}{|c|}{$101.6(213.5-48.4)$} & \multicolumn{3}{|c|}{$138.2(236.5-80.8)$} & \multicolumn{3}{|c|}{73.6 (116.3-46.6) } \\
\hline
\end{tabular}

PPV positive predictive value, NPV negative predictive value, $\mathrm{Cl}$ confidence interval 

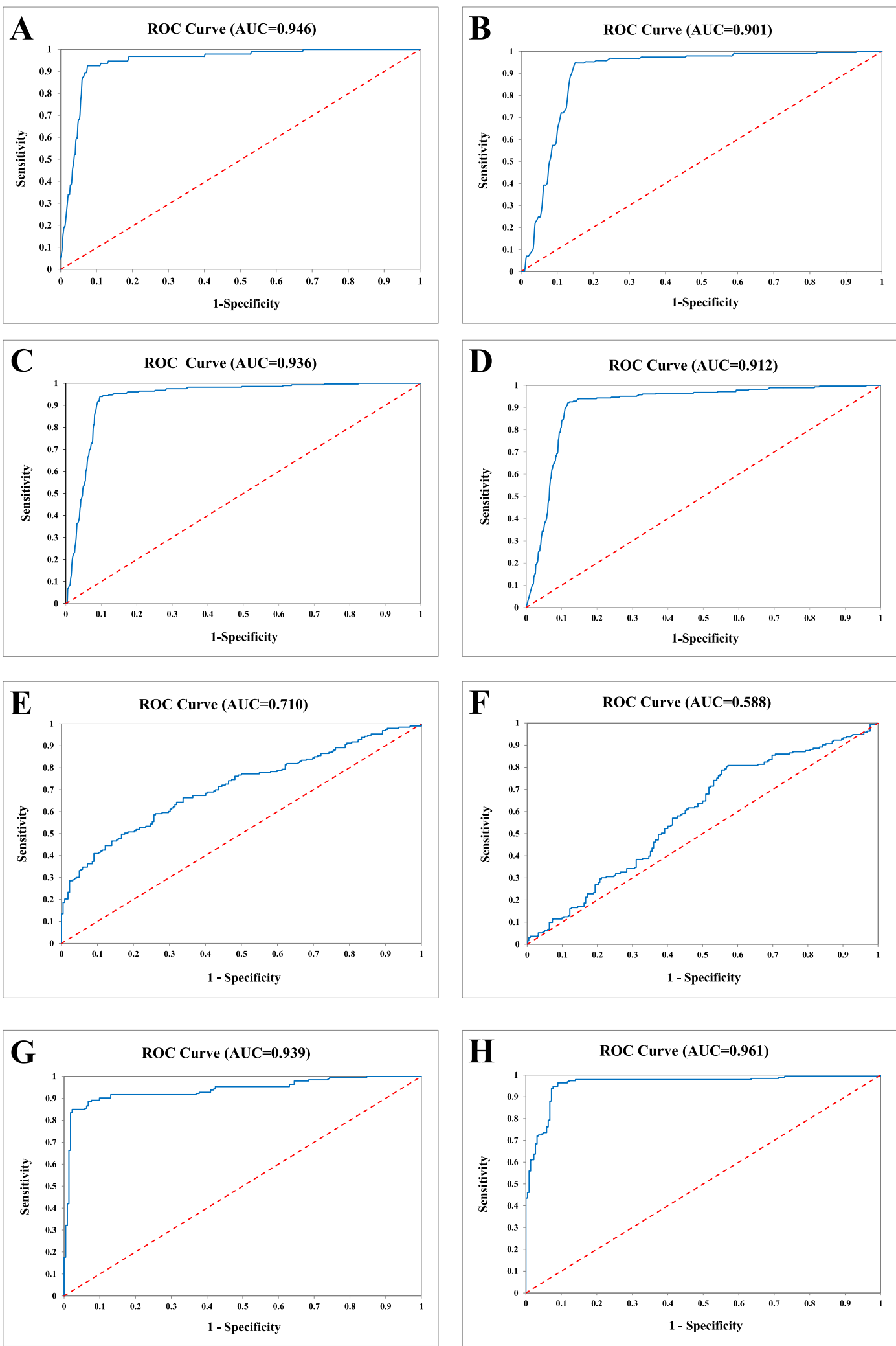

Fig. 2 (See legend on next page.) 
(See figure on previous page.)

Fig. 2 Receiver operating characteristic (ROC) curves for PCa diagnosis. ROC curve of the 25-Gene Panel urine test for PCa diagnosis in the retrospective training cohort (a), in the prospective validation cohort (b), and in the combination cohort (c); ROC curve of cross-validation of the 25-Gene Panel urine test for PCa diagnosis in the combination cohort (d); ROC curve of PSA (e), PSA at 4 ng/mL cutoff (f), the 25-Gene Panel urine test (g), and the 25-Gene Panel urine test and PSA combination (h) for PCa diagnosis in the cohort of 414 patients

test to determine the necessity of subsequent biopsy, the unnecessary biopsies could be reduced by 10-fold to avoid 630,000 biopsies in the USA alone, which could greatly reduce patient suffering and lower medical cost.

In addition, to examine if PSA and the risk factors could be combined with the 25-Gene Panel urine test to enhance its diagnostic performance, various combinations were assessed by multivariate logistic regression analysis. The result showed that when the 25-Gene Panel urine test was combined with PSA (25-Gene+ PSA) in the PSA Cohort, both the odds ratio and AUC were significantly increased (odds ratio of 107.3 (95\% CI 213.2-54.0) and AUC of 0.939 (95\% CI 0.962-0.916) for the 25-Gene Panel alone vs odds ratio of 195.5 (95\% CI 431.4-88.6) and AUC of 0.961 (95\% CI 0.980-0.942) for 25-Gene+PSA) $(p<0.01)$ (Table 4, Fig. 2h). However, combination of the 25-Gene Panel urine test with age (25-Gene+age) in the PSA Cohort, combination of the 25-Gene Panel urine test with family history (25-Gene+ $\mathrm{FH})$ in the FH Cohort, or combination of the 25-Gene Panel urine test with PSA-4 (25-Gene+PSA-4) in the PSA Cohort did not significantly alter the diagnostic accuracy of the 25-Gene Panel urine test, as neither odds ratio nor AUC differ significantly in these combinations (Table 4). Furthermore, important diagnostic measures including sensitivity, specificity, positive predictive value (PPV), and negative predictive value (NPV) of the 25Gene Panel urine test combined with PSA, PSA plus age, PSA-4, and PSA-4 plus age in the PSA Cohort were compared. As shown in Table 5, 25-Gene+PSA had higher accuracy than 25-Gene alone, 25-Gene+PSA-4, or 25-Gene+PSA-4+age, with exceptionally high sensitivity of $94.8 \%$ (95\% CI 98.0-91.7\%), specificity of $91.4 \%$ (95\% CI 95.1-87.8\%), PPV of 90.6\% (95\% CI 94.6-86.6\%), and NPV of $95.3 \%$ (95\% CI 98.2-92.5\%). The addition of age to 25-Gene+PSA did not change its diagnostic accuracy except for a slight increase of AUC (0.961 (95\% CI $0.980-0.942)$ vs 0.967 (95\% CI 0.984-0.950)) ( $p>0.05)$. These results suggest that the 25-Gene Panel urine test can be combined with pre-biopsy PSA to provide more accurate cancer diagnosis.

\section{In silico validation of the 25-Gene Panel for cancer diagnosis}

To validate the differential gene expression of the 25 genes in the panel in $\mathrm{PCa}$ and benign prostate tissue specimens, we used a prostate tissue cohort GSE17951 $(n=154)$ obtained from the GEO database (NCBI) (17, 18). The mRNA expression data of the 25 genes was obtained from the database and normalized with beta-actin expression. A large group of the genes including HIF1A $(p=0.013)$, BIRC5 $(p<0.0001), A M A C R \quad(p<0.0001)$, CRISP3 $(p<0.0001)$, HPN $(p<0.0001)$, MYO6 $(p<$ $0.0001)$, GOLM1 ( $p<0.0001)$, LMTK2 $(p<0.001)$, EZH2 $(p<0.0001)$, PCA3 $(p<0.0001)$, PIP5K1A $(p<0.0001)$, CDK1 $(p<0.0001)$, ANXA3 $(p=0.008)$, CCND1 $(p=$ $0.012)$, and $K L K 3(p<0.0001)$ had significantly increased expression in $\mathrm{PCa}$ specimens as compared with that of benign prostate, while a small group of genes including FGFR1 $(p=0.286)$, TMPRSS2 $(p=0.369)$, VEGFA $(p=$ $0.464)$, and $F N 1$ ( $p=0.632)$ had statistically insignificant increase in gene expression (Additional file 3: Fig. S1). In contrast, several genes including PMP22 $(p<0.0001)$, GSTP1 $(p<0.0001)$, and CST3 $(p<0.0001)$ had significantly decreased expression in PCa specimens as compared with that of benign prostate, and a few genes including CCNA1 $(p=0.112)$, PSCA $(p=0.187)$, and PTEN $(p=0.493)$ had statistically insignificant decrease in gene expression (Additional file 3: Fig. S2). When the 25 genes were combined as a panel, the discriminant score F1 showed strikingly higher level in PCa than that in benign prostate $(p<0.0001)$ (Additional file 3: Fig. S3). In addition, the diagnostic performance of the 25Gene Panel to distinguish PCa and benign prostate was assessed in the GSE17951 cohort and the result showed very high sensitivity of $100 \%$ (95\% CI 100-100\%), specificity of $96.0 \%$ (95\% CI 99.8-92.0\%) (Additional file 2: Table S4), and AUC of 0.998 (95\% CI 1.004-0.992) (Additional file 3: Fig. S4). The in silico study result confirmed the results from the urine study for the high diagnostic accuracy of the 25-Gene Panel.

\section{Identification of clinically significant cancer}

It is important to develop accurate tests to identify and subtype clinically significant and insignificant PCa. We examined whether the 25-Gene Panel urine test could be used to identify clinically significant PCa. In the retrospective and prospective cohorts, 727 patients were diagnosed to have PCa by routine biopsy. Using the 25Gene Panel urine test with a Stratification Algorithm (Additional file 1: Methods), clinically significant and insignificant PCa were identified with high accuracy as shown by AUC of 0.928 (95\% CI 0.947-0.909) (Fig. 3). Such an accurate and convenient urine test may be used 


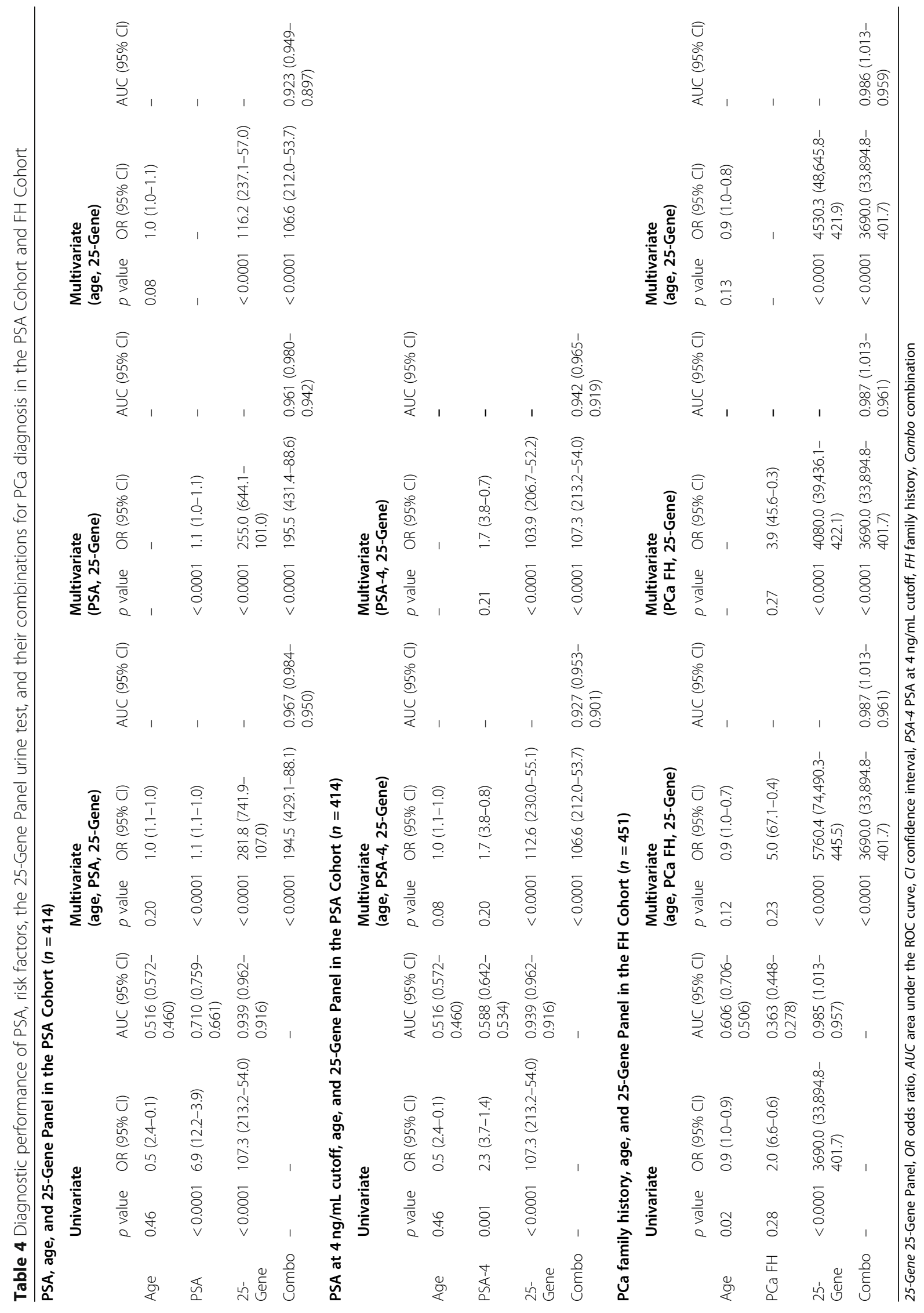


Table 5 Comparison of diagnostic performance of PSA, PSA at $4 \mathrm{ng} / \mathrm{mL}$ cutoff, age, and the $25-G e n e$ Panel urine test and their combinations for PCa diagnosis in the PSA Cohort

\begin{tabular}{lllllll}
\hline & Sensitivity (95\% Cl) & Specificity (95\% Cl) & PPV (95\% Cl) & NPV (95\% Cl) & OR (95\% Cl) & AUC (95\% Cl) \\
\hline PSA & $36.3 \%(43.1-29.5 \%)$ & $92.3 \%(94.8-88.8 \%)$ & $80.5 \%(88.8-72.1 \%)$ & $62.5 \%(67.7-57.3 \%)$ & $6.7(12.2-3.9)$ & $0.710(0.759-0.661)$ \\
PSA-4 & $83.9 \%(89.1-78.8 \%)$ & $30.2 \%(36.2-24.1 \%)$ & $51.1 \%(56.6-45.6 \%)$ & $68.4 \%(77.6-59.2 \%)$ & $2.3(3.7-1.4)$ & $0.588(0.642-0.534)$ \\
Age & $1.0 \%(2.5-0.4 \%)$ & $97.8 \%(99.7-95.8 \%)$ & $28.6 \%(62.0-4.9 \%)$ & $53.3 \%(58.2-48.5 \%)$ & $0.5(2.4-0.1)$ & $0.516(0.572-0.460)$ \\
$25-G e n e$ & $88.6 \%(93.1-84.1 \%)$ & $93.2 \%(96.6-90.0 \%)$ & $91.9 \%(95.9-88.0 \%)$ & $90.4 \%(94.2-86.6 \%)$ & $107.3(213.2-54.0)$ & $0.939(0.962-0.916)$ \\
PSA+25-Gene & $94.8 \%(98.0-91.7 \%)$ & $91.4 \%(95.1-87.8 \%)$ & $90.6 \%(94.6-86.6 \%)$ & $95.3 \%(98.2-92.5 \%)$ & $195.5(431.4-88.6)$ & $0.961(0.980-0.942)$ \\
PSA-4+25-Gene & $88.6 \%(93.1-84.1 \%)$ & $93.2 \%(96.6-90.0 \%)$ & $91.9 \%(95.9-88.0 \%)$ & $90.4 \%(94.2-86.6 \%)$ & $107.3(213.2-54.0)$ & $0.942(0.965-0.919)$ \\
PSA+Age+25-Gene & $94.8 \%(97.9-91.7 \%)$ & $91.4 \%(95.1-87.8 \%)$ & $90.6 \%(94.6-86.5 \%)$ & $95.3 \%(98.2-94.5 \%)$ & $194.5(429.1-88.1)$ & $0.967(0.984-0.950)$ \\
PSA-4+Age+25-Gene & $88.5 \%(93.1-84.0 \%)$ & $93.2 \%(96.6-89.9 \%)$ & $91.9 \%(95.8-88.0 \%)$ & $90.4 \%(94.2-86.6 \%)$ & $106.6(212.0-53.7)$ & $0.927(0.953-0.901)$ \\
\hline
\end{tabular}

PPV positive predictive value, NPV negative predictive value, OR odds ratio, AUC area under the ROC curve, CI confidence interval, PSA-4 PSA at 4 ng/mL cutoff

to identify clinically significant cancer patients for immediate treatment. For patients with clinically insignificant cancer, it can be used periodically to monitor cancer progression during active surveillance.

\section{Preliminary study to test the 25-Gene Panel urine test for prostatectomy treatment follow-up}

Currently, no accurate method is available to check if radical prostatectomy (RP) has completely removed prostate tumors. To test if the 25-Gene Panel urine test could be used to show the absence of PCa after the tumors had been surgically removed by RP, we collected urine from ten patients before and after RP and performed diagnosis using the 25-Gene Panel. As shown in Table 6, nine out of ten urine samples (90\%) were diagnosed to be non-PCa after RP, which was consistent with successful RP in most patients. The one patient diagnosed to be PCa may still have residual cancer lesion after the surgery and need additional treatment. The result of the preliminary study in the small patient cohort suggests that the 25-Gene Panel urine test has potential to be used as an accurate and simple method to measure efficacy of RP for treatment follow-up.

\section{The 25-Gene Panel urine test is PCa-specific}

In the urine cohorts, some patients had other types of cancers in addition to PCa or benign prostate (Table 2), especially urinary tract cancers such as bladder cancer, which might affect $\mathrm{PCa}$ diagnosis since cells of other cancers could be released into the urine. We have not

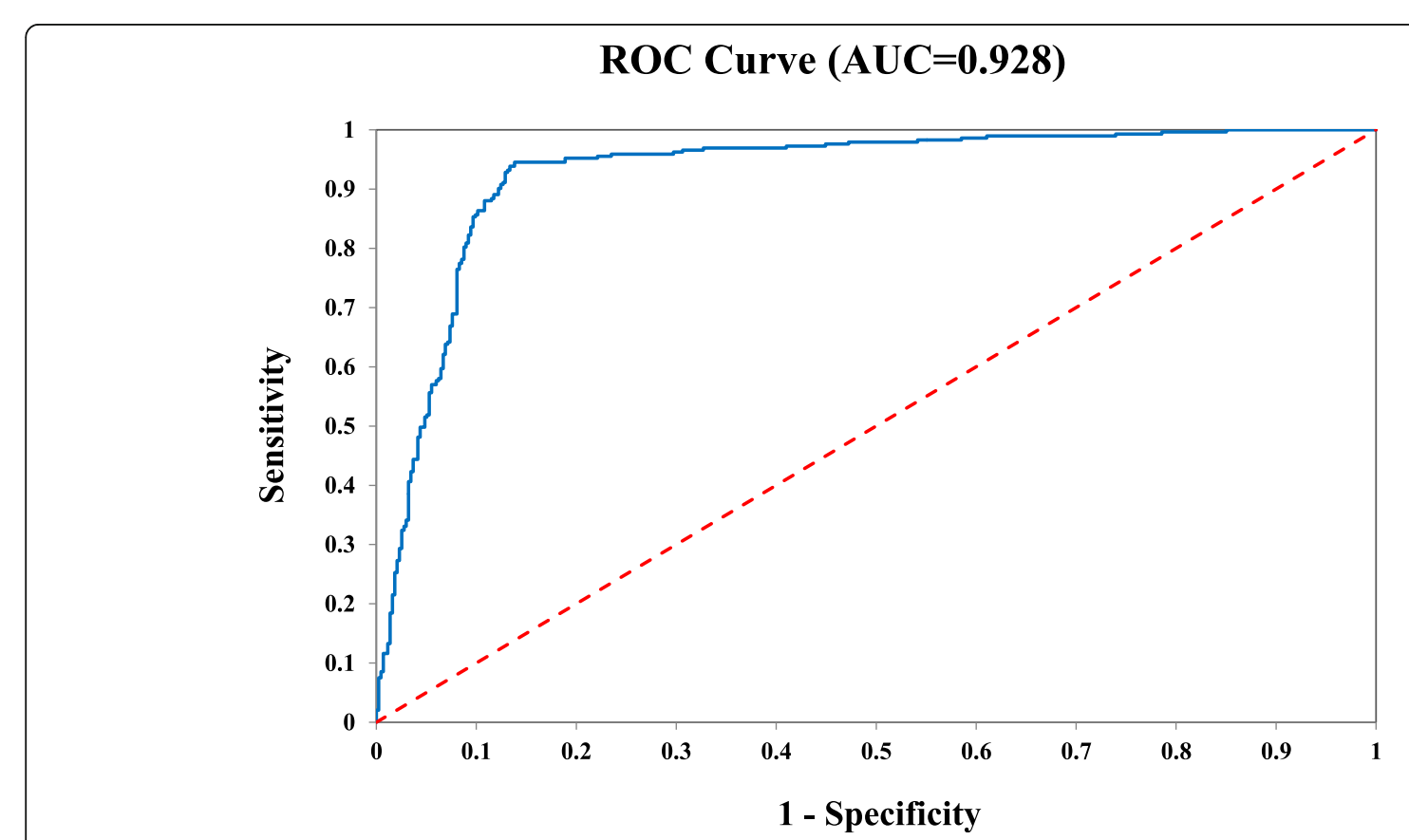

Fig. 3 Receiver operating characteristic (ROC) curve of the 25-Gene Panel urine test for the identification of clinically significant PCa in cancer patients from the retrospective and prospective cohorts $(n=727)$ 
Table 6 Diagnosis of pre- and post-prostatectomy urine samples by the 25-Gene Panel urine test

\begin{tabular}{lll}
\hline & Pre-surgery urine & Post-surgery urine \\
\hline Patient A & PCa & PCa \\
Patient B & PCa & Non-PCa \\
Patient C & PCa & Non-PCa \\
Patient D & PCa & Non-PCa \\
Patient E & PCa & Non-PCa \\
Patient F & PCa & Non-PCa \\
Patient G & PCa & Non-PCa \\
Patient H & PCa & Non-PCa \\
Patient I & PCa & Non-PCa \\
Patient J & PCa & Non-PCa \\
$\%$ Non-PCa & O & $90.0 \%$ \\
\hline
\end{tabular}

found any study addressing this issue; therefore, we tested if the presence of other cancers could affect diagnosis of the 25-Gene Panel urine test. We found that all of the PCa patients who also had other types of cancers (two had bladder cancer, one each had melanoma, kidney and colorectal cancer) were diagnosed to have $\mathrm{PCa}$, while all of the benign prostate patients with other cancers (one had bladder cancer, one each had lung and skin cancer) were diagnosed to be non-PCa. This suggests that our test was specific for $\mathrm{PCa}$ diagnosis without being affected by the presence of other cancers.

\section{Discussion}

In this study, we have developed a novel 25-Gene Panel urine test that can be used for PCa diagnosis to accurately identify patients who need to have biopsy to avoid large amount of unnecessary biopsies each year. In addition, it can be used as an accurate and non-invasive test to identify clinically significant and insignificant cancer to assist treatment decision and active cancer surveillance. Further, it may potentially be used as a treatment follow-up test to assess if residual cancer exists after prostatectomy or other cancer therapies to determine if further treatment is necessary. The 25Gene Panel urine test was found to be specific for PCa diagnosis, even for patients with other types of cancers. Lastly, the non-invasive and convenient urine test without DRE may be performed by patients at home to facilitate cancer surveillance and post-treatment follow-up.

The study population in the retrospective and prospective cohorts represented patients in real clinical practice as they were from the clinical cases obtained from the participating hospitals. These patients with elevated PSA underwent scheduled biopsy for cancer diagnosis/treatment. AUC analysis is an important tool to assess the diagnostic performance of the 25-Gene Panel. In addition, other important parameters including sensitivity, specificity, positive predictive value, negative predictive value, and odds ratio were used to assess the 25-Gene Panel. Thus, combining these measurements provided valid assessment of the 25-Gene Panel urine test.

Currently, none of the clinical parameters (i.e., PSA and its derivatives such as PHI), biomarkers (i.e., PCA3), or combinations of biomarkers or clinical parameters (i.e., PCA3 combined with TMPRSS2:ERG, microRNA signatures, metabolomic biomarkers) used in clinical practice or reported in publications was able to diagnose $\mathrm{PCa}$ or stratify cancer risk with $>90 \%$ sensitivity and specificity, and AUC over 0.9 , as shown in several recent reviews [2, 4-6, 8-10, 25-27]. Our 25-Gene Panel urine test was validated for accurate cancer diagnosis by two independent multi-center study cohorts as well as the large combination cohort with uniformly high diagnostic sensitivity and specificity above $90 \%$ and AUC exceeding 0.9. In statistics, AUC of the ROC curve is an important measure of how accurate a classifier can predict future classification, and AUC over 0.9 indicates an accurate classifier [28]. The fact that the AUC values of the 25Gene Panel urine test in all cohorts were well above 0.9 suggests it may be a more accurate and superior $\mathrm{PCa}$ diagnostic tool than PSA, clinical parameters, existing biomarkers, and their combinations. Our study found that the 25-Gene Panel urine test could be combined with PSA to provide exceptionally accurate diagnosis. In clinical practice, it may be combined with PSA, multiparametric MRI imaging, and biopsy to greatly improve diagnostic accuracy and avoid unnecessary biopsy and overdiagnosis.

For cancer diagnosis and treatment, it is important to identify clinically significant and insignificant cancer so patients with clinically significant cancer are given immediate treatment while clinically insignificant cancer patients are placed under active surveillance. In our study, we found that the 25-Gene Panel was able to accurately identify clinically significant and insignificant cancers. Thus, the 25-Gene Panel has great potential to improve cancer diagnosis and treatment.

In this study, the diagnostic performance of the 25Gene Panel in the retrospective and prospective cohorts were similar, regardless of using freshly collected urine or frozen urine pellet stored for long term. In addition, the PCa patients in the retrospective cohort had a mean PSA level of $6.1 \mathrm{ng} / \mathrm{mL}$, while the patients in the prospective cohort had a high average PSA level of $67.9 \mathrm{ng} /$ $\mathrm{mL}$ (Table 1). This showed that the diagnostic performance of the 25-Gene Panel was not affected by high PSA levels.

The similar diagnostic performance obtained in the cohorts consisting of patients with different ethnic background (Caucasians in the retrospective cohort and 
Asians in the prospective cohort) and clinical characteristics (such as different PSA levels and Gleason scores) suggests that the test is robust and may be used in different patient populations regardless of race, ethnic background, or clinical characteristics.

A small number of urine samples were excluded from the study due to little or no prostate cells collected in the urine. We tested and found that the first morning urine with at least $45 \mathrm{~mL}$ volume, especially the early stream, contained sufficient amount of urine cells for mRNA quantification (data not shown), thus can be used to solve this problem. Since no DRE is necessary and the urine can be stored at room temperature for a week with the DNA/RNA preservative, collecting first morning urine sample is practical for clinical practice. Our noninvasive urine test without DRE that can use urine collected by patients at home represents a novel and significantly improved method for $\mathrm{PCa}$ diagnosis and prognosis.

The 25-Gene Panel consists of several known PCaspecific biomarkers (PCA3, TMPRSS2); biomarkers with potential diagnostic or prognostic values (ANXA3, CRIS P3, CST3, KLK3, PSCA, EZH2, GSTP1, AMACR); biomarkers associated with cellular functions including proliferation, survival, migration, and metastasis (FGFR1, CCNA1, CDK1, CCND1, HIF1A, HPN, VEGFA, PTEN, PIP5K1A); and biomarkers whose involvement in cancer remains unknown (LMTK2, MYO6, BIRC5, FN1, GOLPH2, PMP22) [29-34].

One of the limitations of this study was that there were much less benign prostate urine samples (15.31\%) than PCa urine samples $(84.69 \%)$ in the retrospective cohort, and as a consequence, less benign prostate (28.00\%) than PCa $(72.00 \%)$ samples in the combined cohort. This was due to that less archived benign prostate patient samples were available for our study. The imbalance of the two classes may not reflect the real clinical situation and could theoretically affect the diagnostic measures, resulting in higher sensitivity and PPV, and lower specificity and NPV. However, since the prospective cohort with more balanced benign prostate and $\mathrm{PCa}$ samples $(47.73 \%$ for benign prostate and $52.27 \%$ for $\mathrm{PCa}$ ) had similar diagnostic performance as the retrospective cohort except for higher NPV, it suggests that the effect of the imbalance was limited. Moreover, the AUC of these cohorts were all above 0.9, which suggests that the urine test had similarly high diagnostic accuracy in all cohorts. Nevertheless, it would be better to have a cohort with the number of benign prostate and PCa patients reflecting patient composition in real clinical settings. Thus, more prospective studies will be conducted in the future to further validate the 25-Gene Panel urine test. Another limitation is only a small portion of patients in the retrospective cohort had PSA test result and little cancer staging information was available in the prospective cohort. Thus, large prospective studies with collection of more patient information will be conducted in the future to further validate the 25-Gene Panel urine test and assess its combination with other PCa diagnostic methods such as PSA and MRI imaging. Further, the preliminary study to assess the ability of the 25-Gene Panel urine test to detect if RP has removed cancer lesion was conducted in a small subset of patients who underwent RP, thus future studies with large patient cohorts are needed and will be conducted to determine if the 25-Gene Panel urine test can be used for cancer treatment monitoring.

\section{Conclusions}

In summary, we have developed and validated a highly accurate and non-invasive 25-Gene Panel urine test as the next-generation liquid biopsy method for PCa diagnosis and potential treatment follow-up to improve cancer diagnosis and treatment.

\section{Supplementary Information}

The online version contains supplementary material available at https://doi. org/10.1186/s12916-020-01834-0.

Additional file 1. Supplementary methods, including quantification of mRNA expression, validation of urine test without DRE, Algorithm for identification of clinically significant cancer, and statistical analysis.

Additional file 2: Supplementary tables, including comparison of $\mathrm{Ct}$ values of three genes with and without preamplification before real time qRT-PCR (Table S1), comparison of CtS values of five genes in the urine samples collected with and without digital rectal examination (DRE) (Table S2), genes in the 25-Gene Panel for prostate cancer diagnosis (Table S3), diagnostic performance of the 25-Gene Panel in GSE17951 prostate tissue specimen cohort $(n=154)$ (Table S4)

Additional file 3. Supplementary figures, including box plots of biomarkers with increased gene expression levels in prostate tissue specimens from patients with prostate cancer as compared to patients with benign prostate in the GSE17951 cohort $(n=154)$ (Fig. S1), box plots of biomarkers with decreased gene expression levels in prostate tissue specimens from patients with prostate cancer as compared to patients with benign prostate in the GSE17951 cohort $(n=154)$ (Fig. S2) box plot of discriminant score F1 of the 25-Gene Panel in prostate tissue specimens from patients with prostate cancer as compared to patients with benign prostate in the GSE17951 cohort $(n=154)$ (Fig. S3), and receiver operating characteristic (ROC) curve of the 25-Gene Panel for PCa diagnosis in GSE17951 prostate tissue specimen cohort $(n=154)$ (Fig. S4).

\section{Abbreviations \\ AUC: Area under the curve; CHTN: Cooperative Human Tissue Network; $\mathrm{Cl}$ : Confidence interval; Ct: Cycle threshold; CtS: Relative cycle threshold; DA: Discriminant analysis; DRE: Digital rectal examination; FH: Family history; GEO: Gene Expression Omnibus; IRB: Institutional Review Board; NPV: Negative predictive value; OR: Odds ratio; PCa: Prostate cancer; PPV: Positive predictive value; PSA: Prostate-specific antigen; ROC: Receiver operating curve; RP: Radical prostatectomy}

\section{Acknowledgements}

The authors are grateful to C. Yun for the excellent technical support and W. Zhong and S. Liao for skillful assistance in urine collection. 


\section{Authors' contributions}

$\mathrm{HJ}, \mathrm{LC}, \mathrm{KX}$, and JLP contributed to the study concept and design. $\mathrm{HJ}$ and $\mathrm{AJ}$ contributed to the design and running of the machine learning program. $\mathrm{HJ}$, $\mathrm{HZ}, \mathrm{XF}, \mathrm{CZ}, \mathrm{KX}, \mathrm{AHBW}$, and $\mathrm{LC}$ participated in the study coordination and supervision. JG, TX, FL, and WT contributed to the sample collection. $\mathrm{HJ}, \mathrm{XZ}$, $H Z$, and $X F$ contributed to the sample processing and analysis. $H J, A J, A S$, $N D$, and JLP contributed to the data collection, processing, and statistical analysis. $H J, P A, N D, L K, A S$, and JLP contributed to the data interpretation. $H J, X Z$, and JLP contributed to the literature search. HJ, JG, HZ, KX, JLP, CZ, and $\mathrm{LC}$ contributed to the manuscript writing. The authors read and approved the final manuscript.

\section{Funding}

The authors were funded by The Swedish Cancer Foundation, The Swedish Foundation for Higher Education and Cooperation, Sanming Project of Medicine in Shenzhen (SZSM201412014), The Science and Technology Foundation of Shenzhen (JCYJ20170307095620828, JCYJ20160422145718224, JCYJ20170412155231633, JSGG20170414104216477, and JCYJ20150402152130696), The Shenzhen Urology Minimally Invasive Engineering Centre (GCZX2015043016165448), The Shenzhen Public Service Platform on Tumor Precision Medicine and Molecular Diagnosis, The Shenzhen Cell Therapy Public Service Platform, and Olympia Diagnostics, Inc. The funders had no role in the study design, data collection, and analysis; decision to publish; or preparation of the manuscript. Open Access funding provided by University of Umea.

\section{Availability of data and materials}

The data obtained and analyzed in this study are included in the manuscript.

\section{Ethics approval and consent to participate}

The multi-center retrospective urine study was approved by IRB at San Francisco General Hospital (IRB \# 15-15816) using archived urine samples obtained from Cooperative Human Tissue Network Southern Division and Indivumed $\mathrm{GmbH}$ with appropriate ethical approval and patient consent before urine collection. The multi-center prospective urine study was approved by IRB at Shenzhen People's Hospital (Study Number P2014-006) to collect fresh urine samples from patients treated at seven hospitals collaborated in the study with informed consent.

\section{Consent for publication}

Not applicable.

\section{Competing interests}

Heather Johnson is an employee of Olympia Diagnostics, Inc., and inventor of a pending patent application of prostate cancer diagnostic and prognostic biomarkers. No conflict of interest or financial interest was declared by the other authors.

\section{Author details}

'Olympia Diagnostics, Inc., Sunnyvale, CA, USA. ${ }^{2}$ Department of Urology, The Second Clinical Medical College of Jinan University, Shenzhen People's Hospital, Shenzhen Urology Minimally Invasive Engineering Centre, Shenzhen, China. ${ }^{3}$ Shenzhen Public Service Platform on Tumor Precision Medicine and Molecular Diagnosis, Clinical Medical Research Centre, The Second Clinical College of Jinan University, Shenzhen People's Hospital, Shenzhen, China. ${ }^{4}$ Department of Bio-diagnosis, Institute of Basic Medical Sciences, Beijing, China. ${ }^{5}$ Department of Clinical Pathology and Cytology, Skåne University Hospital, Malmö, Sweden. ${ }^{6}$ Clinical Laboratories, San Francisco General Hospital, San Francisco, CA, USA. ${ }^{7}$ Department of Urology, Foshan First People's Hospital, Foshan, China. ${ }^{8}$ Department of Urology, Nanfang Hospital, Southern Medical University, Guangzhou, China. ${ }^{9}$ Kinetic Reality, Santa Clara, CA, USA. ${ }^{10}$ Department of Translational Medicine, Lund University, Clinical Research Centre, Malmö, Sweden. ${ }^{11}$ Department of Experimental Pathology, Medical University Vienna \& Unit of Laboratory Animal Pathology, University of Veterinary Medicine, Vienna, Austria.

${ }^{12}$ Department of Molecular Biology, Umeå University, 90187 Umeå, Sweden.

${ }^{13}$ Division of Experimental Cancer Research, Department of Translational Medicine, Lund University, 20502 Malmö, Sweden. ${ }^{14}$ Department of Biomedical Sciences, Malmö University, Malmö, Sweden. ${ }^{15}$ Department of Urology, The First Affiliated Hospital of Sun Yat-Sen University, Guangzhou 510080, Guangdong, China.
Received: 4 August 2020 Accepted: 30 October 2020

Published online: 01 December 2020

\section{References}

1. Taitt HE. Global trends and prostate cancer: a review of incidence, detection, and mortality as influenced by race, ethnicity, and geographic location. Am J Mens Health. 2018;12(6):1807-23.

2. Tonry $\mathrm{CL}$, Leacy $\mathrm{E}$, Raso $\mathrm{C}$, et al. The role of proteomics in biomarker development for improved patient diagnosis and clinical decision making in prostate cancer. Diagnostics (Basel). 2016;6(3):27.

3. Botchkina Gl, Kim RH, Botchkina IL, Kirshenbaum A, Frischer Z, Adler HL. Noninvasive detection of prostate cancer by quantitative analysis of telomerase activity. Clin Cancer Res. 2005;11(9):3243-9.

4. Witherspoon L, Breau RH, Lavallée LT. Evidence-based approach to active surveillance of prostate cancer. World J Urol. 2019;6. https://doi.org/10.1007/ s00345-019-02662-5.

5. Osses DF, Roobol MJ, Schoots IG. Prediction medicine: biomarkers, risk calculators and magnetic resonance imaging as risk stratification tools in prostate cancer diagnosis. Int J Mol Sci. 2019; 20(7). doi: https://doi.org/10. 3390/ijms20071637.

6. Raja N, Russell CM, George AK. Urinary markers aiding in the detection and risk stratification of prostate cancer. Transl Androl Urol. 2018;7(Suppl 4): S436-42.

7. Truong M, Yang B, Jarrard DF. Towards the detection of prostate cancer in urine: a critical analysis. J Urol. 2013;189(2):422-9.

8. Matin $F$, Jeet $V$, Moya $L$, et al. A plasma biomarker panel of four microRNAs for the diagnosis of prostate cancer. Sci Rep. 2018;8(1):6653.

9. Kelly RS, Heiden MV, Giovannucci EL, Mucci LA. Metabolomic biomarkers of prostate cancer: prediction, diagnosis, progression, prognosis and recurrence. Cancer Epidemiol Biomark Prev. 2016;25(6):887-906.

10. Fredsøe J, Rasmussen AKI, Thomsen AR, et al. Diagnostic and prognostic microRNA biomarkers for prostate cancer in cell-free urine. Eur Urol Focus. 2018:4(6):825-33.

11. Jamaspishvili T, Kral M, Khomeriki I, et al. Urine markers in monitoring for prostate cancer. Prostate Cancer Prostatic Dis. 2010;13(1):12-9.

12. Carrion DM, Gómez Rivas J, Álvarez-Maestro M, Martínez-Piñeiro L. Biomarkers in prostate cancer management. Is there something new? Arch Esp Urol. 2019;72(2):105-15.

13. Fuessel S, Wirth MP. New markers in prostate cancer: genomics. Arch Esp Urol. 2019;72(2):116-25.

14. Vickers A, Carlsson SV. Toward responsible, informed decision making for prostate cancer treatment decisions. J Clin Oncol. 2019;30:JCO1900989.

15. Boyd LK, Mao X, Lu Y. The complexity of prostate cancer: genomic alterations and heterogeneity. Nature Reviews Urol. 2012;9:652-64.

16. Wang $Y, X i a X Q$, Jia Z, Sawyers A, et al. In silico estimates of tissue components in surgical samples based on expression profiling data. Cancer Res. 2010;70(16):6448-55.

17. Jia Z, Wang $Y$, Sawyers $A$, Yao $H$, et al. Diagnosis of prostate cancer using differentially expressed genes in stroma. Cancer Res. 2011;71(7):2476-87.

18. Kroneis T, Kroneis E, Andersson D, Dolatabadi S, Ståhlberg A. Global preamplification simplifies targeted mRNA quantification. Sci Rep. 2017;7: 45219.

19. Xiao K, Guo J, Zhang X, et al. Use of two gene panels for prostate cancer diagnosis and patient risk stratification. Tumour Biol. 2016;37(8):10115-22.

20. Guo J, Yang J, Zhang X, et al. A panel of biomarkers for diagnosis of prostate cancer using urine samples. Anticancer Res. 2018;38(3):1471-7.

21. Breiman L. Random forests. Machine Learning. 45(1):5-32.

22. Zhao S, Yu J, Wang L. Machine learning based prediction of brain metastasis of patients with IIIA-N2 lung adenocarcinoma by a three-miRNA signature. Transl Oncol. 2018;11(1):157-67.

23. Humphrey PA, Moch H, Cubilla AL, Ulbright TM, Reuter VE. The 2016 WHO classification of tumours of the urinary system and male genital organs-part B: prostate and bladder tumours. Eur Urol. 2016;70(1):106-19.

24. Loeb S, Carter HB, Berndt SI, Ricker W, Schaeffer EM. Complications after prostate biopsy: data from SEER-Medicare. J Urol. 2011;186(5):1830-4.

25. Song $\mathrm{C}$, Chen $\mathrm{H}$, Song $\mathrm{C}$. Research status and progress of the RNA or protein biomarkers for prostate cancer. Onco Targets Ther. 2019;12:2123-36.

26. Neuhaus J, Yang B. Liquid Biopsy Potential Biomarkers in Prostate Cancer. Diagnostics (Basel). 2018;8(4):68. https://doi.org/10.3390/diagnostics8040068.

27. Tomlins SA, Day JR, Lonigro RJ, et al. Urine TMPRSS2:ERG plus PCA3 for individualized prostate cancer risk assessment. Eur Urol. 2016;70(1):45-53. 
28. Pepe MS, Cai T, Longton G. Combining predictors for classification using the area under the receiver operating characteristic curve. Biometrics. 2006; 62(1):221-9.

29. Miftakhova R, Hedblom A, Semenas J, et al. Cyclin A1 and P450 aromatase promote homing and growth of stem-like prostate cancer cells to bone marrow. Cancer Res. 2016;76:2453-64.

30. Galbraith MD, Bender H, Espinosa JM. Therapeutic targeting of transcriptional cyclin-dependent kinases. Transcription. 2019;10(2):118-36.

31. Luo D, Ren H, Zhang W, Xian H, Lian K, Liu H. Clinicopathological and prognostic value of hypoxia-inducible factor-1a in patients with bone tumor: a systematic review and meta-analysis. J Orthop Surg Res. 2019;14(1): 56.

32. Wang K, Peng HL, Li LK. Prognostic value of vascular endothelial growth factor expression in patients with prostate cancer: a systematic review with meta-analysis. Asian Pac J Cancer Prev. 2012:13(11):5665-9.

33. Semenas J, Hedblom A, Miftakhova RR, et al. The role of PI3KAKT-related PIP5K1a and the discovery of its selective inhibitor for treatment of advanced prostate cancer. Proc Natl Acad Sci U S A. 2014;111(35):E3689-98.

34. Jetten $A M$, Suter $U$. The peripheral myelin protein 22 and epithelial membrane protein family. Prog Nucleic Acid Res Mol Biol. 2000;64:97-129.

\section{Publisher's Note}

Springer Nature remains neutral with regard to jurisdictional claims in published maps and institutional affiliations.

Ready to submit your research? Choose BMC and benefit from:

- fast, convenient online submission

- thorough peer review by experienced researchers in your field

- rapid publication on acceptance

- support for research data, including large and complex data types

- gold Open Access which fosters wider collaboration and increased citations

- maximum visibility for your research: over $100 \mathrm{M}$ website views per year

At $\mathrm{BMC}$, research is always in progress.

Learn more biomedcentral.com/submissions 